\title{
Structural convergence for tubulin binding of CPAP and vinca domain microtubule inhibitors
}

Valérie Campanacci ${ }^{1}$, Agathe Urvoas ${ }^{1}$, Liza Ammar Khodja ${ }^{1}$, Magali Aumont-Nicaise ${ }^{1}$, Magali Noiray ${ }^{1}$, Sylvie Lachkar ${ }^{2}$, Patrick A. Curmi ${ }^{3}$, Philippe Minard ${ }^{1}$, Benoît Gigant ${ }^{1, \#}$

${ }^{1}$ Université Paris-Saclay, CEA, CNRS, Institute for Integrative Biology of the Cell (I2BC), 91198, Gif-sur-Yvette, France.

${ }^{2}$ Centre de Recherche des Cordeliers, INSERM U1138, Team "Metabolism, Cancer \& Immunity", Sorbonne Université, Université de Paris, Institut Universitaire de France, Paris, France.

${ }^{3}$ Patrick A. Curmi, Université Paris-Saclay, Inserm, Univ Evry, Structure-Activité des Biomolécules Normales et Pathologiques, 91025, Evry, France.

\# Correspondence to benoit.gigant@i2bc.paris-saclay.fr(BG).

\begin{abstract}
Microtubule dynamics is regulated by various cellular proteins and perturbed by small molecule compounds. To what extent the mechanism of the former resembles that of the latter is an open question. We report here structures of tubulin bound to the PN2-3 domain of CPAP, a protein controlling the length of the centrioles. We show that an $\alpha$-helix of the PN2-3 N-terminal region binds and caps the longitudinal surface of the tubulin $\beta$ subunit. Moreover, a PN2-3 N-terminal stretch lies in a $\beta$-tubulin site also targeted by fungal and bacterial peptide-like inhibitors of the vinca domain, sharing a very similar binding mode with these compounds. Therefore, our results identify several characteristic features of cellular partners that bind to this site and highlight a structural convergence of CPAP with small molecules inhibitors of microtubule assembly.
\end{abstract}

\section{Introduction}

Microtubules are eukaryotic dynamic filaments made of $\alpha \beta$-tubulin heterodimers and involved in major cellular functions including ciliogenesis, intracellular transport and cell division. The dynamics of microtubules is regulated by several families of proteins ${ }^{1}$. It is also perturbed by many small molecule compounds that target a growing number of tubulin binding sites ${ }^{2,3}$. Some 
of these compounds have proven to be useful to fight human diseases, in particular in oncology 4,5 .

Among these microtubule dynamics inhibitors, a diverse set of molecules including vinca alkaloids ${ }^{6}$ and short peptides or depsipeptides ${ }^{7}$ interact with the so-called tubulin vinca domain. This site is located at the interface between two tubulin molecules arranged in a curved protofilament-like manner ${ }^{3,8,9}$. These compounds therefore interact with the longitudinal surface of both $\alpha$ - and $\beta$-tubulin. Doing so, they prevent protofilaments from adopting the straight conformation observed in the microtubule core, which accounts for their microtubule assembly inhibition. Whereas the $\alpha$-tubulin subsite of vinblastine also binds to the $\mathrm{N}$-terminal $\beta$-hairpin motif of stathmin proteins ${ }^{9}$, whether cellular tubulin regulators similarly bind to the vinca domain pocket of the $\beta$ subunit is not known.

Centrosomal P4.1-associated protein (CPAP, also named CENPJ) is a 1338-residue long protein originally identified as a partner of the cytoskeletal protein $4.1 \mathrm{R}-135^{10}$. CPAP is however best characterized as a protein involved in centriole length regulation, its overexpression leading to abnormally long centriolar microtubules ${ }^{11-15}$. This activity can be recapitulated by a minimal construct comprising the tubulin-binding domain of CPAP, named PN2-3 ${ }^{16}$, a microtubule-binding domain which is C-terminal to PN2-3 ${ }^{17}$ and a dimerization motif ${ }^{12}$. PN2-3, which prevents microtubule assembly when isolated, was initially delineated as the CPAP $311-422$ region ${ }^{16}$ and subsequently refined to a fragment of $\sim 76$ residues ${ }^{18}$. Using a tubulin-binding DARPin protein ${ }^{19}$ as a crystallization helper, a partial structure of PN2-3 bound to tubulin was reported ${ }^{12,14}$. Because the N-terminal part of PN2-3 competes with the DARPin for tubulin binding, this region was not visible in the crystal structure, and only about 15 residues of the PN2-3 C-terminal region, defined as the SAC domain ${ }^{12}$, were traced bound to a $\beta$-tubulin surface that corresponds to the outer surface of the microtubule.

Here, we report structures of tubulin bound to CPAP constructs as ternary complexes with $\alpha$ tubulin specific $\alpha$ Rep artificial proteins ${ }^{20,21}$. We show that part of the PN2-3 N-terminal region interacts with the $\beta$-tubulin longitudinal surface in a way very similar to that of vinca domain peptide ligands, therefore defining the type of cellular partners that binds to this site.

\section{Results and Discussion}

Two structures of tubulin-bound PN2-3 define the interaction of this protein with tubulin.

We took advantage of the recently selected $\alpha$-tubulin specific $\alpha$ Rep proteins ${ }^{20}$ to obtain two different crystal forms of $\alpha$ Rep-tubulin-CPAP ternary complexes. The CPAP constructs used 
were based on the 321-397 fragment (Fig. 1A). First, we determined a $2.7 \AA$ resolution structure (Table S1; crystal form 1, with one complex per asymmetric unit) in which the CPAP residues 339 to 387 could be traced (Fig. 1B). These crystals were obtained with the iiH5 $\alpha$ Rep. Surprisingly, in addition to the previously described iiH5-tubulin interaction ${ }^{20}$, we identified a second tubulin-binding site for the $\alpha$ Rep partner (Fig. 1B). In this structure, the CPAP 339360 region folds as an $\alpha$-helix which is connected by a linker, which does not interact with tubulin, to the C-terminal 371-387 SAC domain (Fig. 1B). Accordingly, the linker residues are among the least conserved ones of the CPAP PN2-3 domain from vertebrates (Fig. 1A). This linker is nevertheless reasonably defined in our structure, being stabilized by crystal contacts (see below).

To enhance the likelihood to obtain other crystal forms, we replaced the linker region by a shorter 5-glycine motif (Fig. 1A). We checked by ITC the effect of this modification on the binding affinity for tubulin. Interestingly, the resulting " $\triangle$ loop" CPAP construct displayed an affinity for tubulin slightly higher than the unmodified protein $\left(\mathrm{K}_{\mathrm{D}} 8 \mathrm{nM}\right.$ vs $53 \mathrm{nM}$; Fig. 1C, Fig. S1) and led to a high affinity complex. A new crystal form (Table S1; crystal form 2, two complexes per asymmetric unit), diffracting X-rays up to $2.35 \AA$ resolution, was then obtained using the iE5 $\alpha$ Rep crystallization chaperone. Remarkably, in this case too, two $\alpha$ Rep molecules were bound per tubulin, identifying a second iE5 binding site in addition to the one previously described ${ }^{20}$ (Fig. 1D). In this structure, the 5-glycine linker was disordered. The PN2-3 N-terminal $\alpha$-helix was also less well defined than in crystal form 1, its two C-terminal turns being disordered. In contrast, the region N-terminal to this helix became visible from residues Asn321 to Ala330, whereas residues 331 to 338 remained poorly defined. In the next sections, the information from the two structures will be combined for analysis.

\section{PN2-3 wraps around $\beta$-tubulin.}

Using $\alpha$ Rep proteins as crystallization helpers, we were able to trace the entire region of PN2-3 predicted to interact with tubulin ${ }^{18}$. This region comprises three modules, an N-terminal stretch, an $\alpha$-helix and the SAC domain, that effectively interact with tubulin. They are separated by two non-interacting modules, a "bulge" and the linker (Fig. 1A, 2A,B). The bulge is poorly ordered, but the other modules are clearly defined in one structure or the other. PN2-3 wraps around the tubulin $\beta$ subunit, with the stretch and $\alpha$-helix N-terminal moiety that caps a $\beta$ tubulin surface which is engaged in longitudinal interactions within protofilaments. The bulge allows the N-terminal stretch to be oriented about $90^{\circ}$ to the $\alpha$-helix and to point toward the lumen of the microtubule when PN2-3 is modeled at the plus end of a protofilament (Fig. 2A). 
Overall, these results provide a complete structural view of the binding of PN2-3 to tubulin, which agrees with previous in vitro competition and cross-linking experiments ${ }^{12,14,18}$. They also provide a structural explanation for the inhibition of microtubule assembly by PN2-3. In its longest form, the PN2-3 N-terminal $\alpha$-helix encompasses residues 339 to 360, in good agreement with the NMR-determined helical region of isolated PN2-3 ${ }^{18}$. It binds in a groove at the tip of $\beta$-tubulin boxed in by loop T5 and the H11-H11' region (Fig. 2B), giving a rationale for the competition for tubulin binding with the DARPin used as a crystallization helper in previous structural studies ${ }^{12,14}$ (Fig. 2C). It also explains the binding competition with maytansine ${ }^{12,22}$ (Fig. 2C). Only the N-terminal moiety of this CPAP $\alpha$-helix interacts with tubulin. This part is the only one that is ordered in crystal form 2 (Fig. 2B). In addition to being two turns shorter, the $\alpha$-helix in this crystal form is also rotated $\sim 19^{\circ}$ compared to crystal form 1 (best viewed in Fig. 2A). These observations argue for a moderate contribution of the CPAP $\mathrm{N}$-terminal $\alpha$-helix to the interaction with tubulin, in agreement with the mild effect of mutations in this helix on the binding affinity ${ }^{14}$.

\section{The N-terminal stretch of PN2-3 targets the binding site of vinca domain inhibitors.}

The N-terminal stretch of the $\Delta$ loop construct in crystal form 2 (residues 321 to 330 of CPAP) interacts with tubulin in an extended conformation, mostly without forming regular secondary structure apart a short $3{ }_{10}$ helix from residues 322 to 324 . It binds in a site mainly contributed by helix H1, loop T5, and the H6-H7 region of the $\beta$ subunit. It also interacts with the M-loop residue Arg278 and with GDP (Fig. 3A). This last interaction together with those involving T5 and the $\mathrm{H} 7$ residue Tyr $224^{8}$ accounts for the inhibition by CPAP of tubulin nucleotide exchange 18. Therefore, PN2-3 contacts the same $\beta$-tubulin structural elements as small molecule compounds binding to the vinca domain. Strikingly, the PN2-3 N-terminal stretch closely overlaps with peptide and depsipeptide microtubule inhibitors, including phomopsin A, auristatins and tubulysins, four structural features being shared by CPAP and most of these compounds ${ }^{23}$. They are described hereunder.

First, the highly conserved Ile327 CPAP residue (Fig. 1A) points in a hydrophobic pocket of $\beta$-tubulin boxed in by Val177 (from T5), Tyr210 (H6), Pro222 (H6-H7 loop), Tyr224 and Leu227 (H7) (Fig. 3B). We tested the effect of an I327R substitution in the 321-397 construct and found a $\sim 5$-fold decrease of the binding affinity for tubulin (Fig. 3C, Fig. S1C,F). This tubulin pocket is targeted by all the vinca domain ligands whose interaction with tubulin has been structurally characterized (Fig. 3D,E, Fig. S2). Remarkably, it accommodates a small aliphatic group in all cases, and it has been shown that replacing the corresponding isopropyl 
group in tubulysins by a bulkier aryl or cyclohexyl one leads to inactive compounds ${ }^{24}$. Accordingly, modeling indicates that replacing Ile327 with a Phe residue in the tubulin-CPAP $\Delta$ loop structure would lead to steric conflicts with neighboring tubulin residues.

A second feature is the proline residue adjacent to Ile327 (Pro326, also conserved). A proline or proline-like residue is found at this position or at an adjacent one in most of the peptide-like inhibitors of tubulin (Fig. S2). Thirdly, whereas the Arg325 guanidino group interacts with the nucleotide base (Fig. 3A), its side chain aliphatic part together with Ile322 forms a hydrophobic patch interacting with $\beta$-tubulin, including with Tyr224. This tyrosine is in a stacking interaction with the guanine of the nucleotide and is involved in the regulation of microtubule assembly ${ }^{25}$. Whereas the hydrophobic nature of the tubulin region interacting with these two CPAP residues is not strong, the observation that the vinca domain peptide inhibitors all have a hydrophobic group superposing with Ile322 and with the aliphatic part of Arg325 (Fig. 3E, Fig. S2) suggests such groups are important for tubulin binding. Accordingly, removing the corresponding phenyl group in tubulysin D leads to a compound $\sim 40$-fold less efficient in inhibiting cancer cell growth ${ }^{26}$.

Finally, two acidic CPAP residues (Glu323 and Glu324) are in positions to make salt bridges with Lys19 and Arg278 of $\beta$-tubulin, respectively (Fig. 3F), suggesting that these glutamic acid residues contribute substantially to the interaction with tubulin. This proposal is supported by the observation that several small peptide-like compounds also have acidic groups overlapping with Glu323 and Glu324 (Fig. 3E, Fig. S2), and the interaction with the M-loop residue Arg278 has been proposed to enhance the affinity of some inhibitors for tubulin ${ }^{27}$. In addition, several compounds shown to also bind to the "partial" binding site of the tubulin-stathmin complex, contributed by $\beta$-tubulin only, have one or two acidic groups (e.g. phomopsin $\mathrm{A}^{8}$ ). On the other hand, replacing the acidic group in tubulysins with, e.g., an hydrazine counterpart has only a mild effect on the activity ${ }^{28,29}$. In the case of CPAP, an E323R-E324R mutant displayed an affinity for tubulin similar to that of the wild type construct (Fig. 3C, Fig. S1). Consistently, the affinity of a 3R mutant, having in addition the I327R substitution, was similar to that of the single I327R mutant (Fig. S1E,F). It should also be noted that, whereas Glu323 is a conserved CPAP residue, Glu324 is not (Fig. 1A). We therefore conclude that Glu323 and Glu324 do not contribute much to the binding affinity of PN2-3 for tubulin.

Interestingly, whereas the N-terminal stretch of the 321-397 construct was not visible in crystal form 1 , the vinca domain peptide site is not empty in that structure but occupied by the linker (which connects the N-terminal $\alpha$-helix to the SAC domain) of a crystal-related symmetry 
molecule. Remarkably, the ${ }^{362}$ EGPL $^{365}$ motif of the linker in that crystal form fills the space occupied by ${ }^{324} \mathrm{ERPI}^{327}$ in crystal form 2 (Fig. 3G). This observation accounts for the destabilized N-terminal stretch in crystal form 1. More importantly, it confirms features important for targeting the vinca domain peptide of tubulin (Fig. S2).

\section{Conclusion.}

In this study, we have shown that the PN2-3 domain of CPAP interacts with tubulin through three discrete, mostly linear binding motifs, a feature of intrinsically disordered proteins ${ }^{30,31}$. Another characteristic of this category of proteins is their remaining dynamicity even when bound to their partners. Our results, which identify two conformations for the N-terminal $\alpha$-helix and an $\mathrm{N}$-terminal stretch that is defined in only one crystal form, indeed suggest that PN2-3 retains some mobility when bound to tubulin. In addition, the overall affinity for tubulin is likely to come from the cumulative effect of the three interacting motifs, since constructs including either the $\mathrm{N}$-terminal stretch and the N-terminal $\alpha$-helix or the C-terminal SAC domain display $\mathrm{K}_{\mathrm{D}}$ in the $\mu \mathrm{M}$ to the tens of $\mu \mathrm{M}$ range, i.e. two to three orders of magnitude higher than that of PN2-3 ${ }^{12,14}$. Such a binding mode, characterized by a dynamic complex and a cumulative contribution of weakly interacting points to the affinity, is likely to accommodate the mutation of (a priori) important residues (e.g. Ile327), as evidenced by the moderate effect of these mutations on the affinity.

To conclude, we have determined the complete structure of the microtubule destabilizing motif of the CPAP protein bound to tubulin. We have shown that its N-terminal stretch interacts with the vinca domain of $\beta$-tubulin. Its binding mode is very similar to that of several vinca domain peptide-like compounds from bacteria or fungi, which inhibit microtubule assembly. This finding points to a structural convergence for tubulin binding and provides constraints on the type of cellular partners accommodated in this pocket.

\section{Methods.}

Proteins. The CPAP 321-397 construct was obtained by standard molecular biology methods from the PN2-3 DNA and inserted in the pET3d plasmid. $\Delta$ loop and point mutants were then generated from this construct by PCR. All constructs were verified by sequencing. The corresponding proteins were overexpressed in the BL21(DE3) E. coli strain and purified as described for PN2-3 ${ }^{18}$. Tubulin was purified from sheep brain ${ }^{32}$. The iE5 and iiH5 $\alpha$ Reps were produced and purified as described previously ${ }^{20}$. 
Isothermal titration calorimetry (ITC). Calorimetric experiments were conducted at $20^{\circ} \mathrm{C}$ with a MicroCal PEAK-ITC instrument (Malvern). All proteins were buffer-exchanged to $20 \mathrm{mM}$ Mes-K pH 6.8, $1 \mathrm{mM} \mathrm{MgCl2,0.01} \mathrm{mM} \mathrm{EGTA} \mathrm{and} \mathrm{0.01} \mathrm{mM} \mathrm{GDP.} \mathrm{Aliquots} \mathrm{(2} \mu \mathrm{l})$ of CPAP constructs at $150 \mu \mathrm{M}$ concentration were injected into a $15 \mu \mathrm{M}$ tubulin solution (cell volume, $0.24 \mathrm{ml}$ ). Analysis of the data was performed using the MicroCal PEAK-ITC software provided by the manufacturer according to the one-binding-site model. The Origin software (Malvern) was used to superpose the data shown in Fig. 1C and 3C.

Crystallization and structure determination. The $\alpha$ Rep-tubulin-CPAP construct complexes were concentrated to about $20 \mathrm{mg} \mathrm{ml}^{-1}$ for crystallization experiments. iiH5-tubulin-CPAP 321-397 construct (crystal form 1) was crystallized at $293 \mathrm{~K}$ by vapor diffusion in a crystallization buffer consisting of $0.1 \mathrm{M}$ Mes-K pH 6.8, $8 \%$ (W/V) polyethylene glycol 3350. To obtain these crystals, the lysine residues of the 321-397 construct were methylated prior to the formation of the complex ${ }^{33}$. In addition, residue Leu342 had been mutated to a methionine to help tracing the CPAP chain in lower resolution data using SeMet version of this construct at earlier stages of this project. Crystals were harvested in the crystallization buffer containing also $20 \%$ glycerol and flash-cooled in liquid nitrogen. iE5-tubulin-CPAP $\Delta$ loop construct crystals (crystal form 2) were obtained at $293 \mathrm{~K}$ in $0.18 \mathrm{M}$ tri-ammonium citrate, $0.1 \mathrm{M}$ Mes$\mathrm{K}$ pH 6.8, $18.5 \%(\mathrm{~W} / \mathrm{V})$ polyethylene glycol 3350 and cryoprotected in mother liquor supplemented with $20 \%$ glycerol. Datasets were collected at $100 \mathrm{~K}$ at the SOLEIL Synchrotron (PROXIMA-1 and PROXIMA-2A beamlines). Crystal form 1 data were processed with XDS ${ }^{34}$ as implemented in the XDSME package ${ }^{35}$. Data for crystal form 2 were processed with autoPROC 36 which implements the STARANISO treatment for anisotropy (http://staraniso.globalphasing.org/). Structures were solved by molecular replacement with Phaser ${ }^{37}$ using tubulin-iiH5 (pdb id 6GWD) and tubulin-iE5 (pdb id 6GWC) as search models, and refined with BUSTER ${ }^{38}$ with iterative model building in Coot ${ }^{39}$. Data collection and refinement statistics are reported in Table S1. Figures of structural models were generated with PyMOL (www.pymol.org).

Sequence conservation. The sequence conservation of the CPAP PN2-3 domain (Fig. 1A) was calculated using the ConSurf program ${ }^{40}$. The analysis was based on 150 sequences retrieved from the UniProt database and having a maximum of 95\% sequence identity with human PN23 taken as a reference. About $40 \%$ of the sequences were from mammals, $55 \%$ from birds and $5 \%$ from reptiles, and there was one amphibian CPAP.

Accession codes. Coordinates and structure factors have been deposited with the Protein Data Bank with accession numbers 7Q1E (crystal form 1) and 7Q1F (crystal form 2). 


\section{Acknowledgements}

We thank M. Knossow (I2BC, Gif-sur-Yvette) for many useful discussions and for a critical reading of the manuscript. Diffraction data were collected at SOLEIL synchrotron (PROXIMA1 and PROXIMA-2A beamlines, Saint-Aubin, France). We are most grateful to the machine and beam line groups for making these experiments possible. This work has benefited from the crystallization and protein interaction platforms of I2BC supported by French Infrastructure for Integrated Structural Biology (FRISBI) ANR-10-INBS-05. Financial support by CNRS and by the Fondation ARC pour la Recherche sur le Cancer (Grant PJA20161204544 to B.G.) is acknowledged.

\section{References}

(1) Desai, A.; Mitchison, T. J. Microtubule Polymerization Dynamics. Annu Rev Cell Dev Biol 1997, 13, 83-117. https://doi.org/10.1146/annurev.cellbio.13.1.83.

(2) Mühlethaler, T.; Gioia, D.; Prota, A. E.; Sharpe, M. E.; Cavalli, A.; Steinmetz, M. O. Comprehensive Analysis of Binding Sites in Tubulin. Angew. Chem. Int. Ed Engl. 2021. https://doi.org/10.1002/anie.202100273.

(3) Steinmetz, M. O.; Prota, A. E. Microtubule-Targeting Agents: Strategies to Hijack the Cytoskeleton. Trends Cell Biol. 2018, 28 (10), 776-792. https://doi.org/10.1016/j.tcb.2018.05.001.

(4) Amos, L. A. What Tubulin Drugs Tell Us about Microtubule Structure and Dynamics. Semin. Cell Dev. Biol. 2011, 22 (9), 916-926. https://doi.org/10.1016/j.semcdb.2011.09.014.

(5) Risinger, A. L.; Giles, F. J.; Mooberry, S. L. Microtubule Dynamics as a Target in Oncology. Cancer Treat. Rev. 2009, 35 (3), 255-261. https://doi.org/10.1016/j.ctrv.2008.11.001.

(6) Duflos, A.; Kruczynski, A.; Barret, J.-M. Novel Aspects of Natural and Modified Vinca Alkaloids. Curr. Med. Chem. Anti-Cancer Agents 2002, 2 (1), 55-70. https://doi.org/10.2174/1568011023354452.

(7) Hamel, E.; Covell, D. G. Antimitotic Peptides and Depsipeptides. Curr. Med. Chem. AntiCancer Agents 2002, 2 (1), 19-53. https://doi.org/10.2174/1568011023354263.

(8) Cormier, A.; Marchand, M.; Ravelli, R. B. G.; Knossow, M.; Gigant, B. Structural Insight into the Inhibition of Tubulin by Vinca Domain Peptide Ligands. EMBO Rep. 2008, 9 (11), 1101-1106. https://doi.org/10.1038/embor.2008.171.

(9) Gigant, B.; Wang, C.; Ravelli, R. B.; Roussi, F.; Steinmetz, M. O.; Curmi, P. A.; Sobel, A.; Knossow, M. Structural Basis for the Regulation of Tubulin by Vinblastine. Nature 2005, 435 (7041), 519-522. https://doi.org/10.1038/nature03566.

(10) Hung, L. Y.; Tang, C. J.; Tang, T. K. Protein 4.1 R-135 Interacts with a Novel Centrosomal Protein (CPAP) Which Is Associated with the Gamma-Tubulin Complex. Mol. Cell. Biol. 2000, 20 (20), 7813-7825. https://doi.org/10.1128/mcb.20.20.7813-7825.2000.

(11) Kohlmaier, G.; Lončarek, J.; Meng, X.; McEwen, B. F.; Mogensen, M. M.; Spektor, A.; Dynlacht, B. D.; Khodjakov, A.; Gönczy, P. Overly Long Centrioles and Defective Cell Division upon Excess of the SAS-4-Related Protein CPAP. Curr. Biol. 2009, 19 (12), 1012-1018. https://doi.org/10.1016/j.cub.2009.05.018.

(12) Sharma, A.; Aher, A.; Dynes, N. J.; Frey, D.; Katrukha, E. A.; Jaussi, R.; Grigoriev, I.; Croisier, M.; Kammerer, R. A.; Akhmanova, A.; Gönczy, P.; Steinmetz, M. O. Centriolar CPAP/SAS4 Imparts Slow Processive Microtubule Growth. Dev. Cell 2016, 37 (4), 362-376. https://doi.org/10.1016/j.devcel.2016.04.024.

(13) Tang, C.-J. C.; Fu, R.-H.; Wu, K.-S.; Hsu, W.-B.; Tang, T. K. CPAP Is a Cell-Cycle Regulated Protein That Controls Centriole Length. Nat. Cell Biol. 2009, 11 (7), 825-831. 
https://doi.org/10.1038/ncb1889.

(14) Zheng, X.; Ramani, A.; Soni, K.; Gottardo, M.; Zheng, S.; Ming Gooi, L.; Li, W.; Feng, S.; Mariappan, A.; Wason, A.; Widlund, P.; Pozniakovsky, A.; Poser, I.; Deng, H.; Ou, G.; Riparbelli, M.; Giuliano, C.; Hyman, A. A.; Sattler, M.; Gopalakrishnan, J.; Li, H. Molecular Basis for CPAP-Tubulin Interaction in Controlling Centriolar and Ciliary Length. Nat. Commun. 2016, 7, 11874. https://doi.org/10.1038/ncomms11874.

(15) Schmidt, T. I.; Kleylein-Sohn, J.; Westendorf, J.; Clech, M. L.; Lavoie, S. B.; Stierhof, Y.-D.; Nigg, E. A. Control of Centriole Length by CPAP and CP110. Curr. Biol. 2009, 19 (12), 1005-1011. https://doi.org/10.1016/j.cub.2009.05.016.

(16) Hung, L.-Y.; Chen, H.-L.; Chang, C.-W.; Li, B.-R.; Tang, T. K. Identification of a Novel Microtubule-Destabilizing Motif in CPAP That Binds to Tubulin Heterodimers and Inhibits Microtubule Assembly. Mol. Biol. Cell 2004, 15 (6), 2697-2706. https://doi.org/10.1091/mbc.e04-020121.

(17) Hsu, W.-B.; Hung, L.-Y.; Tang, C.-J. C.; Su, C.-L.; Chang, Y.; Tang, T. K. Functional Characterization of the Microtubule-Binding and -Destabilizing Domains of CPAP and d-SAS-4. Exp. Cell Res. 2008, 314 (14), 2591-2602. https://doi.org/10.1016/j.yexcr.2008.05.012.

(18) Cormier, A.; Clément, M.-J.; Knossow, M.; Lachkar, S.; Savarin, P.; Toma, F.; Sobel, A.; Gigant, B.; Curmi, P. A. The PN2-3 Domain of Centrosomal P4.1-Associated Protein Implements a Novel Mechanism for Tubulin Sequestration. J. Biol. Chem. 2009, 284 (11), 6909-6917. https://doi.org/10.1074/jbc.M808249200.

(19) Pecqueur, L.; Duellberg, C.; Dreier, B.; Jiang, Q.; Wang, C.; Plückthun, A.; Surrey, T.; Gigant, B.; Knossow, M. A Designed Ankyrin Repeat Protein Selected to Bind to Tubulin Caps the Microtubule plus End. Proc Natl Acad Sci USA 2012, 109 (30), 12011-12016. https://doi.org/10.1073/pnas.1204129109.

(20) Campanacci, V.; Urvoas, A.; Consolati, T.; Cantos-Fernandes, S.; Aumont-Nicaise, M.; Valerio-Lepiniec, M.; Surrey, T.; Minard, P.; Gigant, B. Selection and Characterization of Artificial Proteins Targeting the Tubulin $\alpha$ Subunit. Structure 2019, 27 (3), 497-506.

https://doi.org/10.1016/j.str.2018.12.001.

(21) Chevrel, A.; Mesneau, A.; Sanchez, D.; Celma, L.; Quevillon-Cheruel, S.; Cavagnino, A.; Nessler, S.; Li de la Sierra-Gallay, I.; van Tilbeurgh, H.; Minard, P.; Valerio-Lepiniec, M.; Urvoas, A. Alpha Repeat Proteins (ARep) as Expression and Crystallization Helpers. J. Struct. Biol. 2018, 201 (2), 88-99. https://doi.org/10.1016/j.jsb.2017.08.002.

(22) Prota, A. E.; Bargsten, K.; Diaz, J. F.; Marsh, M.; Cuevas, C.; Liniger, M.; Neuhaus, C.; Andreu, J. M.; Altmann, K.-H.; Steinmetz, M. O. A New Tubulin-Binding Site and Pharmacophore for Microtubule-Destabilizing Anticancer Drugs. Proc. Natl. Acad. Sci. 2014, 111 (38), 13817-13821. https://doi.org/10.1073/pnas.1408124111.

(23) Li, W.; Tan, L.; Zhang, Z.; Xia, Q.; Lei, D.; Li, Y.; Zhang, T.; Zeng, S.; Sima, X.; Wang, Y. The X-Ray Structure of Tubulysin Analogue TGL in Complex with Tubulin and Three Possible Routes for the Development of next-Generation Tubulysin Analogues. Biochem. Biophys. Res. Commun. 2021, 565, 29-35. https://doi.org/10.1016/j.bbrc.2021.05.086.

(24) Shankar, S. P.; Jagodzinska, M.; Malpezzi, L.; Lazzari, P.; Manca, I.; Greig, I. R.; Sani, M.; Zanda, M. Synthesis and Structure-Activity Relationship Studies of Novel Tubulysin U Analogues Effect on Cytotoxicity of Structural Variations in the Tubuvaline Fragment. Org. Biomol. Chem. 2013, 11 (14), 2273-2287. https://doi.org/10.1039/C3OB27111K.

(25) Ayukawa, R.; Iwata, S.; Imai, H.; Kamimura, S.; Hayashi, M.; Ngo, K. X.; Minoura, I.; Uchimura, S.; Makino, T.; Shirouzu, M.; Shigematsu, H.; Sekimoto, K.; Gigant, B.; Muto, E. GTPDependent Formation of Straight Tubulin Oligomers Leads to Microtubule Nucleation. J Cell Biol 2021, 220 (e202007033), e202007033. https://doi.org/10.1083/jcb.202007033.

(26) Patterson, A. W.; Peltier, H. M.; Sasse, F.; Ellman, J. A. Design, Synthesis, and Biological Properties of Highly Potent Tubulysin D Analogues. Chem. - Eur. J. 2007, 13 (34), 9534-9541. https://doi.org/10.1002/chem.200701057.

(27) Waight, A. B.; Bargsten, K.; Doronina, S.; Steinmetz, M. O.; Sussman, D.; Prota, A. E. Structural Basis of Microtubule Destabilization by Potent Auristatin Anti-Mitotics. PLOS ONE 2016, 11 (8), e0160890. https://doi.org/10.1371/journal.pone.0160890.

(28) Leamon, C. P.; Reddy, J. A.; Vetzel, M.; Dorton, R.; Westrick, E.; Parker, N.; Wang, Y.; 
Vlahov, I. Folate Targeting Enables Durable and Specific Antitumor Responses from a Therapeutically Null Tubulysin B Analogue. Cancer Res. 2008, 68 (23), 9839-9844. https://doi.org/10.1158/0008-5472.CAN-08-2341.

(29) Colombo, R.; Wang, Z.; Han, J.; Balachandran, R.; Daghestani, H. N.; Camarco, D. P.; Vogt, A.; Day, B. W.; Mendel, D.; Wipf, P. Total Synthesis and Biological Evaluation of Tubulysin Analogues. J. Org. Chem. 2016, 81 (21), 10302-10320. https://doi.org/10.1021/acs.joc.6b01314.

(30) Wright, P. E.; Dyson, H. J. Linking Folding and Binding. Curr. Opin. Struct. Biol. 2009, 19 (1), 31-38. https://doi.org/10.1016/j.sbi.2008.12.003.

(31) Yang, J.; Gao, M.; Xiong, J.; Su, Z.; Huang, Y. Features of Molecular Recognition of Intrinsically Disordered Proteins via Coupled Folding and Binding. Protein Sci. 2019, 28 (11), 19521965. https://doi.org/10.1002/pro.3718.

(32) Castoldi, M.; Popov, A. V. Purification of Brain Tubulin through Two Cycles of Polymerization-Depolymerization in a High-Molarity Buffer. Protein Expr. Purif. 2003, 32 (1), 8388. https://doi.org/10.1016/S1046-5928(03)00218-3.

(33) Nawrotek, A.; Guimarães, B. G.; Velours, C.; Subtil, A.; Knossow, M.; Gigant, B. Biochemical and Structural Insights into Microtubule Perturbation by CopN from Chlamydia Pneumoniae. J. Biol. Chem. 2014, 289 (36), 25199-25210. https://doi.org/10.1074/jbc.M114.568436. (34) Kabsch, W. XDS. Acta Crystallogr. D Biol. Crystallogr. 2010, 66 (Pt 2), 125-132. https://doi.org/10.1107/S0907444909047337.

(35) Legrand, P. XDSME: XDS Made Easier. GitHub Repository. DOI 10.5281/Zenodo.837885. 2017.

(36) Vonrhein, C.; Flensburg, C.; Keller, P.; Sharff, A.; Smart, O.; Paciorek, W.; Womack, T.; Bricogne, G. Data Processing and Analysis with the AutoPROC Toolbox. Acta Crystallogr. D Biol. Crystallogr. 2011, 67 (4), 293-302. https://doi.org/10.1107/S0907444911007773.

(37) McCoy, A. J.; Grosse-Kunstleve, R. W.; Adams, P. D.; Winn, M. D.; Storoni, L. C.; Read, R. J. Phaser Crystallographic Software. J. Appl. Crystallogr. 2007, 40 (Pt 4), 658-674.

https://doi.org/10.1107/S0021889807021206.

(38) Bricogne, G.; Blanc, E.; Brandl, M.; Flensburg, C.; Keller, P.; Paciorek, W.; Roversi, P.; Sharff, A.; Smart, O.; Vonrhein, C.; Womack, T. BUSTER Version 2.10.3. Cambridge, United Kingdom: Global Phasing Ltd. 2017.

(39) Emsley, P.; Lohkamp, B.; Scott, W. G.; Cowtan, K. Features and Development of Coot. Acta Crystallogr. D Biol. Crystallogr. 2010, 66 (Pt 4), 486-501.

https://doi.org/10.1107/S0907444910007493.

(40) Ashkenazy, H.; Abadi, S.; Martz, E.; Chay, O.; Mayrose, I.; Pupko, T.; Ben-Tal, N. ConSurf 2016: An Improved Methodology to Estimate and Visualize Evolutionary Conservation in Macromolecules. Nucleic Acids Res. 2016, 44 (W1), W344-350. https://doi.org/10.1093/nar/gkw408.

\section{Figure legends.}

Figure 1. Overview of tubulin-CPAP structures. (A) Constructs used in this study and sequence conservation of the PN2-3 domain of CPAP. The PN2-3 sequence (residues 311-422 of CPAP, with an additional alanine after the $\mathrm{N}$-terminal methionine) is aligned with that of constructs used in crystallization experiments. These constructs are based on the CPAP 321-397 region and include an additional valine after the N-terminal methionine. The residues 321 to 387 were traced in the different structures. This region has been divided into 5 structural motifs, as indicated under the sequence. Residues that have been mutated for affinity measurements are highlighted in yellow in the sequence of the 321-397 construct. The PN2-3 sequence conservation scores range from 1 (not conserved, blue) to 9 (highly conserved, magenta) according to the Consurf color code. (B) Crystal form 1: structure of the 321-397 CPAP 
fragment (cyan) in complex with tubulin (dark and light grey). Tubulin is further bound to two molecules (orange and yellow) of the iiH5 $\alpha$ Rep used as a crystallization helper. (C) Isothermal titration calorimetry (ITC) of the interaction between tubulin and the 321-397 CPAP fragment or the $\Delta$ loop construct (see also Fig. S1A,B,F). (D) Crystal form 2: structure of tubulin bound to the CPAP $\Delta$ loop fragment (magenta). The complex was crystallized with two molecules of the iE5 $\alpha$ Rep. In panels $B$ and $D$, the $\alpha$ Reps in orange are those bound to tubulin in two structures of tubulin- $\alpha$ Rep previously determined ${ }^{20}$, the ones in yellow define new binding sites.

Figure 2. PN2-3 wraps around $\beta$-tubulin. (A) PN2-3 (cyan and magenta, from crystal forms 1 and 2, respectively) is modeled at the plus end of a microtubule (pdb id 6DPU), by superposing $\beta$-tubulin of tubulin-CPAP complexes to $\beta$-tubulin at the plus end of a protofilament. View from the plus end of the microtubule, three protofilaments are shown and CPAP is modeled on the middle one. The five structural motifs of PN2-3 are indicated (see also panel B and Fig. 1A). (B) The PN2-3 N-terminal $\alpha$-helix binding site. The $\beta$-tubulin elements interacting with this $\alpha$-helix are highlighted in green. (C) Structural basis for PN2-3 competition with a DARPin (pdb id 5EIB) and with maytansine (4TV8) for tubulin binding. The side chain of Gln345, which would overlap with maytansine, is shown.

Figure 3. The interaction of the N-terminal stretch of PN2-3 with tubulin. (A) Overview of the interaction. The PN2-3 $\Delta$ loop construct (from crystal form 2) is in magenta, and the tubulininteracting elements are in green. The side chain of CPAP Lys328 which is weakly defined in the electron density maps has been truncated after its $C \beta$ atom. (B) Zoom in on the interaction with tubulin of the CPAP residue Ile327. (C) ITC analysis of the tubulin:I327R and tubulin:E323R-E324R interaction (see also Fig. S1C,D,F). (D,E) The binding sites of vinblastine (pdb id 1Z2B, panel D) and of phomopsin A (3DU7, panel E) overlap with that of the N-terminal stretch of PN2-3. (F) Zoom in on the interaction with tubulin of the CPAP residues Glu323 and Glu324. (G) In crystal form 1, the linker region of a symmetry-related CPAP molecule (cyan) occupies the tubulin binding site of the N-terminal stretch of PN2-3. 
A

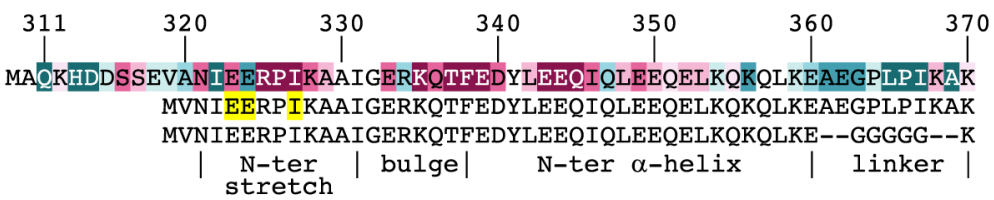
PN2-3
CPAP ( 321-397)

$\triangle 100 p$

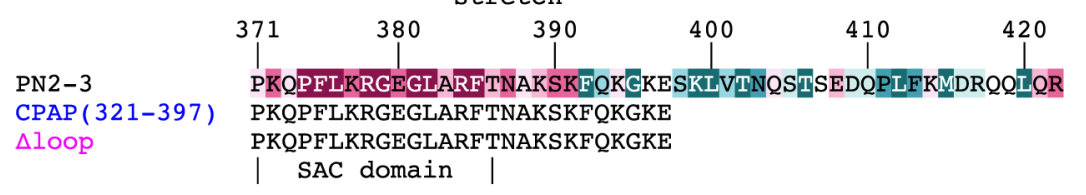

Consurf code:

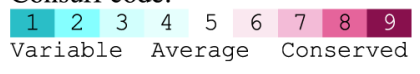

B

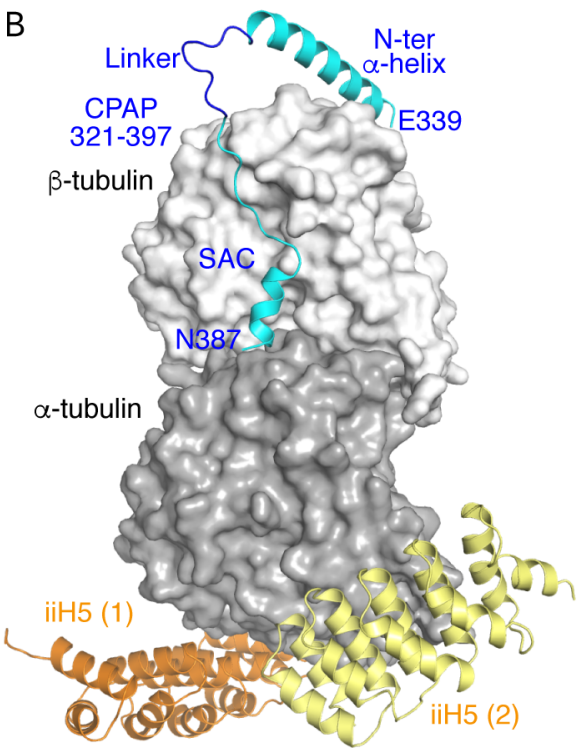

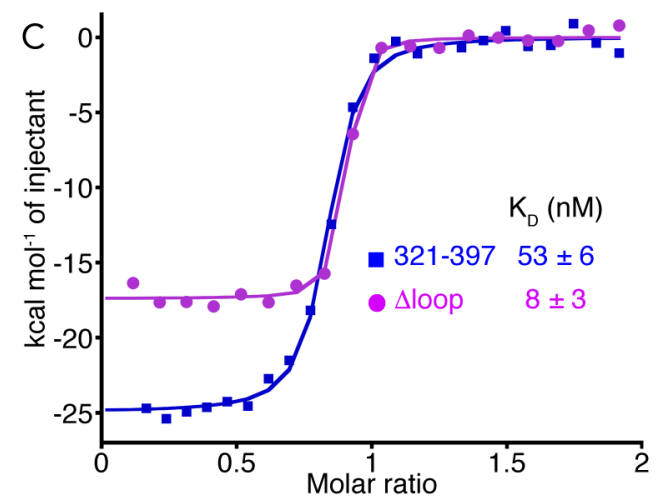

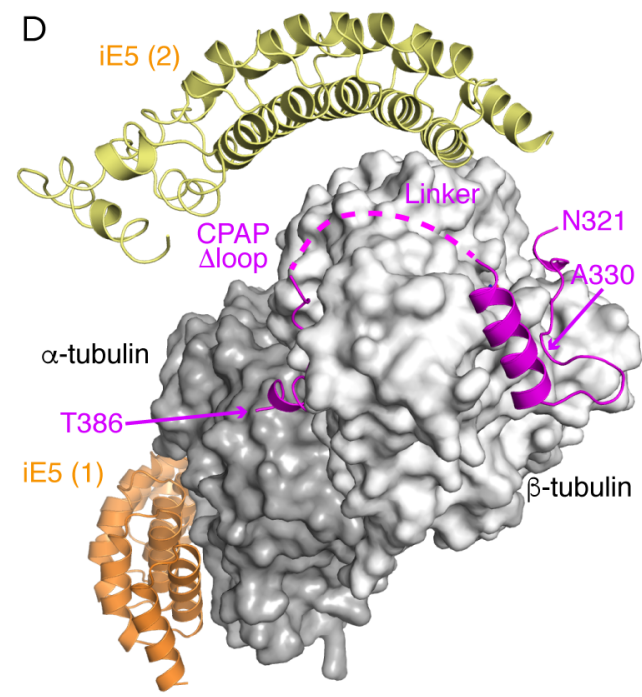

Figure 1 
bioRxiv preprint doi: https://doi.org/10.1101/2021.10.19.464980; this version posted October 21, 2021. The copyright holder for this preprint (which was not certified by peer review) is the author/funder. All rights reserved. No reuse allowed without permission.

A

CPAP: crystal form 1

crystal form 2

Outside
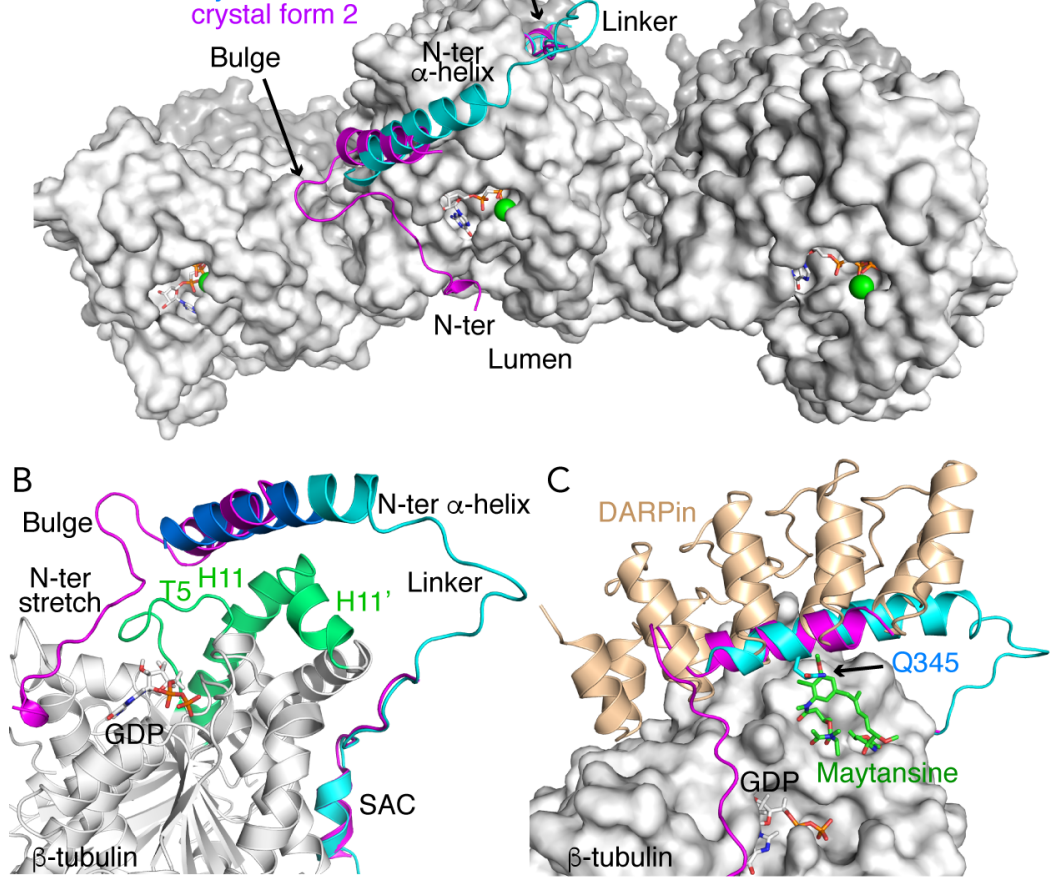

Figure 2 
bioRxiv preprint doi: https://doi.org/10.1101/2021.10.19.464980; this version posted October 21, 2021. The copyright holder for this preprint (which was not certified by peer review) is the author/funder. All rights reserved. No reuse allowed without permission.

A
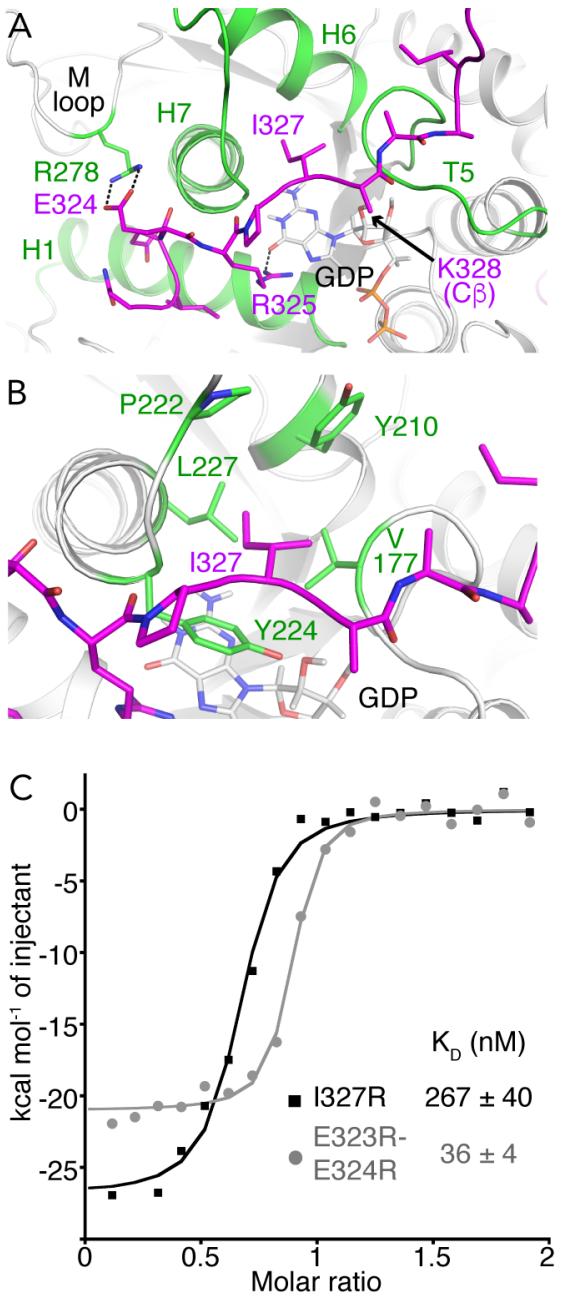

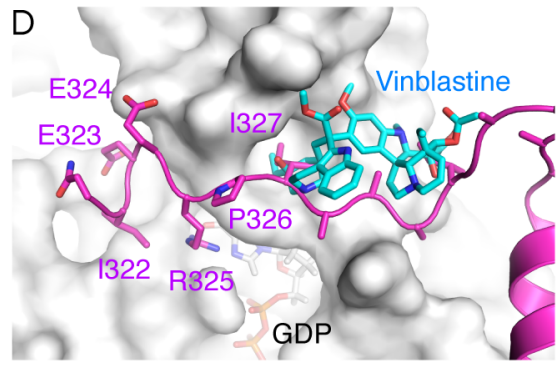

E
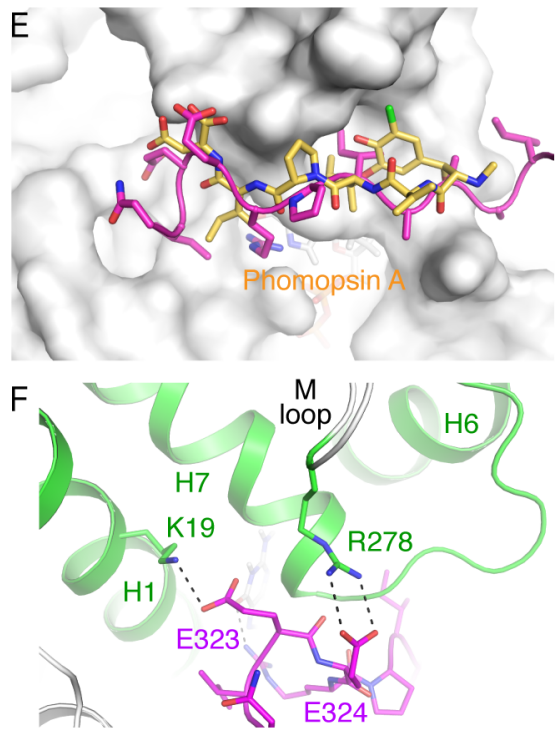

G crystal form 2 crystal form 1 (symmetric)

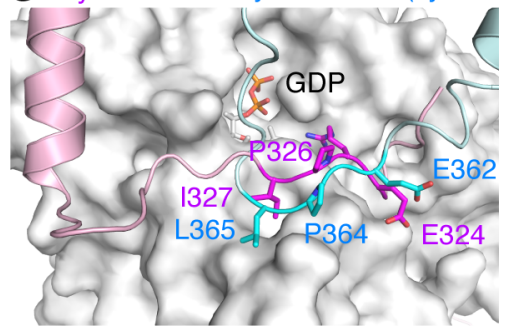

Figure 3 